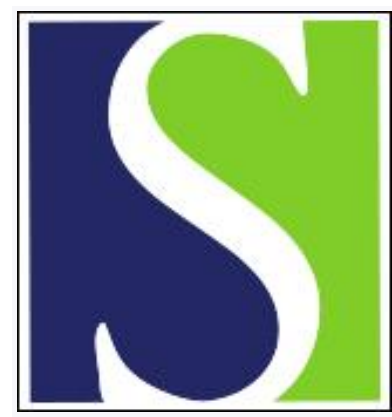

Scand J Work Environ Health 2013;39(5):468-476

https://doi.org/10.5271/sjweh.3359

Published online: 25 Mar 2013, Issue date: 01 Sep 2013

Work-related psychosocial and mechanical risk factors for work disability: a 3-year follow-up study of the general working population in Norway

by Sterud T

The evidence of work-related mechanical and psychosocial risk factors for work disability is limited. This study provides evidence of a substantial relationship between both work-related mechanical and psychosocial factors and work disability in the general working population. Work disability prevention interventions could focus on monotonous and physically demanding jobs involving prolonged standing, neck flexion, and whole-body vibration.

Affiliation: National Institute of Occupational Health, Oslo, Norway PO Box 8149 Dep, No-0033 Oslo, Norway. tom.sterud@stami.no

Refers to the following texts of the Journal: 1997;23(6):403-413

2002;28(4):222-231 1994;20(1):65-66 2011;37(4):276-287

2013;39(2):125-133 2013;39(4):351-360

The following articles refer to this text: 2017;43(5):405-414;

2022;48(2):137-147

Key terms: biomechanical exposure; disability; disability pension; job control; job demand; job exposure; mechanical; mechanical risk factor; Norway; prospective study; psychosocial; psychosocial risk factor; stress; work disability; workload; workplace

This article in PubMed: www.ncbi.nlm.nih.gov/pubmed/23529701

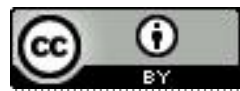




\title{
Work-related psychosocial and mechanical risk factors for work disability: a 3-year follow-up study of the general working population in Norway
}

\author{
by Tom Sterud, $P h D^{1}$
}

\begin{abstract}
Sterud T. Work-related psychosocial and mechanical risk factors for work disability: a 3-year follow-up study of the general working population in Norway. Scand J Work Environ Health. 2013;39(5):468-476. doi:10.5271/sjweh.3359

Objectives This study examines the impact of work-related psychosocial and mechanical risk factors for work disability in the general working population.

Methods A randomly drawn cohort from the general population in Norway aged 18-66 years was followed for 3 years ( $\mathrm{N}=12550,67 \%$ response rate at baseline). Eligible respondents were in paid work for $\geq 10$ hours per week in 2006 and were still in paid work or had quit working because of health problems (work disability) in 2009 (N=6745). Five work-related psychosocial factors and eight mechanical exposures were measured. The outcome of interest was self-reported work disability at 3-year follow-up.
\end{abstract}

Results In total, 2.6\% (176 individuals) reported work disability at the 3-year follow-up. Disability rates were higher among women, older workers, and those with fewer years of education and higher levels of psychological distress and musculoskeletal complaints. After adjusting for these factors, work-related psychosocial predictors of disability were low levels of supportive leadership [odds ratio (OR) 1.61, 95\% confidence interval (95\% CI) 1.02-2.56] and monotonous work (OR 1.53, 95\% CI 1.09-2.16). Mechanical factors were neck flexion (OR 2.49, 95\% CI 1.36-4.56), prolonged standing (OR 1.79, 95\% CI 1.21-2.46), whole-body vibration (OR 4.15, 95\% CI $1.77-9.71$ ), and heavy physical work (OR 2.23. 95\% CI 1.08-4.57). The estimated population risk attributable to these factors was about $45 \%$.

Conclusion Monotonous work, prolonged standing, neck flexion, and whole-body vibration appear to be the most consistent and important predictors of work disability.

Key terms biomechanical exposure; disability pension; job control; job demand; job exposure; prospective study; stress; workload; workplace.

The inability to work because of sickness and disability generate considerable costs to the individual as well as society. OECD statistics show that, on average, $6 \%$ of the working-age population is on disability benefits; in Norway, the disability rate is close to $10 \%$ (1). However, disability rates vary considerably between occupations (2), and more knowledge is needed about the impact of specific risk factors at the workplace.

Occupational disability is usually the result of a long process of sickness absence relating to deteriorating health and failing ability to cope with the demands of work. In addition to work-related mechanical exposure, work-related psychosocial factors have been acknowledged as potential risk factors. Several studies have shown that heavy physical work is associated with increased risk of disability (3-5). Other mechanical fac- tors like prolonged standing (6), whole body vibration (7), and work in uncomfortable positions (8) have been discussed as possible risk factors, but the evidence still remains limited (9). Low job control at work (eg, little influence on the work situation and decision authority) appears to be the most consistent work-related psychosocial risk factor (10-14). Some studies have indicated that job strain $(15,16)$, lack of social support $(8,17)$, and job demands (14) may be important. However, other studies have not supported these associations $(18,19)$.

Despite an increase in the number of studies on the relationship between exposure to work-related risk factors and work disability, assessment of the impact of work on disability remains a topic of great interest. Firstly, the effects of multiple factors are supported by evidence from few studies, and the consistency across

1 National Institute of Occupational Health, Oslo, Norway.

Correspondence to: Tom Sterud, National Institute of Occupational Health, Oslo, Norway PO Box 8149 Dep, NO-0033 Oslo, Norway. [E-mail: tom.sterud@stami.no] 
studies is low. Secondly, several studies have argued that psychosocial factors may be confounded by mechanical exposures at work and vice versa $(20,21)$, yet there are few large prospective studies on general working populations with a sufficient variety of both mechanical and psychosocial factors to distinguish between their effects on work disability $(18,19)$.

The aim of the present study was to identify workrelated psychosocial and mechanical factors that contribute to the risk of work disability. We simultaneously tested the impact of psychosocial and mechanical factors in work settings in a randomly selected three-year prospective cohort in the general working population in Norway. Potential confounders such as age, sex, education, psychological distress, and musculoskeletal complaints were taken into account.

\section{Methods}

\section{Study design and study population}

Data were provided from the nationwide study of living conditions-work environment conducted by Statistics Norway (SSB). Data were collected during two periods (18 September 2006-24 February 2007 and 22 June 2009-09 January 2010) by personal telephone interviews $(0.5 \%$ of the completed interviews were face-toface interviews). Prior to telephone contact, potential respondents were informed by mail about the study, the topic of the study, and data/privacy protection.

Eligible respondents were community-living Norwegian residents aged 18-66 years. In 2006, a gross sample of 18679 was randomly drawn from this population. Of these, 12550 persons were interviewed (67\%). The baseline sample was compared to the gross sample according to the benchmarks of age, gender, and region. No major differences were detected (22). The cohort data, that is those who participated in the survey in both 2006 and 2009, consisted of 9371 persons (response rate $=50.2 \%$ of the gross sample $/ 74.6 \%$ of the baseline sample). Respondents who were in paid work for $\geq 10$ hours in $2006(\mathrm{~N}=9621)$ and were still in paid work in $2009(\mathrm{~N}=6572)$ or had quit working due to health problems $(\mathrm{N}=176)$ constituted the follow-up sample in the present paper $(\mathrm{N}=6748)$.

In order to address the issue of possible selection bias related to dropouts between baseline and follow-up, we compared background characteristics, exposure levels, and the level of health complaints (eg, neck/shoulder pain, low-back pain, and psychological distress) in the baseline and follow-up samples. In the follow-up sample, there was a slight under-representation of the youngest and oldest respondents. There were no gender differences in the two samples, but there was a slight under-representation of people with a low level of education. Correspondingly, there was a slight underrepresentation of "service workers and shop and market sales workers", "plant and machine operators and assemblers" and people working in "elementary occupations" (23). Furthermore, the results show that the level of exposure and the prevalence of health complaints were nearly identical across the two samples. The associations between different types of psychosocial and mechanical exposure and the health variables were also very similar across the two samples.

\section{Measurement}

Psychosocial measures. The variable job demands was measured by a single item, developed by Statistics Norway: "Do you sometimes have so much to do that you have to skip lunch, work longer than your normal working hours, or take work home with you?" (answer categories: every day, a couple of days a week, one day a week, a couple of days a month, and less often or not at all). Monotonous work was measured by a single item: "Does your job consist of constantly repeated tasks, meaning that you do the same thing hour after hour?" (answer categories: almost the whole time, around three-quarters of the work-day, half of the work-day, a quarter of the work-day, and seldom or never). Other dimensions were measured with scales from the General Nordic Questionnaire (QPSNordic) for psychological and social factors at work (24). Job control was measured with three items $(\alpha=0.69)$ : (i) "To what extent can you decide the pace at which you work?" (ii) "To what extent can you influence decisions that are important to your work?" and (iii) "To what extent are you free to decide how to go about doing your work?" Supportive leadership was also measured with three items $(\alpha=0.71)$ : (i) "If needed, how often can you get support and help from your immediate superior with your work?" (ii) "Does your immediate superior appreciate your achievements at work?" and (iii) "Does your immediate superior treat employees fair and impartially?" The items were scored on a 5-point scale (very seldom or never, seldom, sometimes, rather often, and quite often or always). Each factor was computed as the mean of valid responses, and the 5-point-scales were converted into three categories: low (1-2), medium (2.1-3), and high (3.1-5). Bullying/harassment was measured with three items: "Do you sometimes: (Q1) receive unwanted sexual attention, comments, etc. at your workplace? (Q2) get bothered or teased in an unpleasant way by your colleagues? (Q3) get bothered or teased in an unpleasant way by superiors?" (answer categories: yes, $\geq 1$ a week; yes, $\geq 1$ a month; no). Bullied/harassed was dichotomized into exposed for the ones who answered "yes" on any of the three questions. 
Mechanical exposure at work. Perceived mechanical workload was measured with eight items developed by an expert group in a Nordic cooperation project (25): (i) "Are you, in your day-to-day work, exposed to vibrations that cause your whole body to shake, eg, from a tractor, forklift truck or other piece of machinery?" (whole-body vibration); (ii) "Do you work with your hands raised to shoulder height or higher?" (Hands above shoulder height); (iii) "Do you have to lift things in uncomfortable positions?" (awkward lifting); (iv) "Do you need to squat or kneel in the course of your work?" (squatting/kneeling); (v) "Do you work with your head bent forward?" (neck flexion); (vi) "Do you work standing up?" (standing) and (vii) "Does your work involve so much effort that it causes you to breathe more rapidly?" (heavy physical work). The response categories were yes or no. "Yes" respondents were asked to estimate the proportion of the working day during which they were exposed (response categories: almost the whole time, $3 / 4$ of the workday, $1 / 2$ of the workday, $1 / 4$ of the workday, and very little of the workday). Scores were categorized into "none or very little of the workday", " $1 / 4$ of the workday", " $1 / 2$ of the workday" and " $3 / 4$ of the workday or more". (viii) Heavy lifting was measured with a single item: "Do have to lift anything that weighs $>20 \mathrm{~kg}$ on a daily basis?" (Response categories: $\geq 20$ times a day, 5-19 times; 1-4 times; no).

Confounder measures. Psychological distress was measured with two questions: "Have you during the past month been very bothered, quite bothered, slightly bothered or not bothered by: (Q1) dejection or depression? or (Q2) nervousness, anxiety or restlessness?" Psychological distress was coded into three categories "not afflicted", "slightly afflicted", and "somewhat or severely afflicted" on either of the two questions. Musculoskeletal complaints were measured with two items: "Have you over the past month been severely afflicted, somewhat afflicted, a little afflicted, or not afflicted at all by: (Q1) pain in your neck or shoulders? or (Q2) pain in the small or lower part of the back?" Occupation was based on an open questionnaire and coded by Statistics Norway into professional title in accordance with the International Standard Classification of Occupations (ISCO-88) and recoded into ten occupational groups. Educational level was based on administrative register data and was coded into five educational levels. Gender and age was based on selfreported information (see table 1).

Outcome. The outcome measure work disability was based on the following questions: (i) "Which year did you stop doing the job that you had last time you were interviewed (2006)?" and (ii) "What was the most important reason for you leaving your last job?" Cases were defined as respondents who reported they were no longer employed due to own health problems / own disease at follow-up (work disability).

\section{Statistical analysis}

The associations between work environment and work disability were calculated as odds ratios (OR) with $95 \%$ confidence intervals $(95 \% \mathrm{CI})$. Missing data was handled by list-wise deletion.

Logistic regression analyses adjusted for potential confounders. Model 1 adjusted for gender and age. In the fully adjusted model 2, further adjustments were made for educational level, psychological distress, musculoskeletal complaints, and work-related predictors. To limit the potential for over-adjustment, each work-related predictor was adjusted only for other workrelated predictors that were first estimated to exert an influence above a certain threshold level. This estimation was made a priori based on the following procedure suggested by Rothman et al (26) applied to baseline data. In the first step, crude OR were separately estimated for each work-related factor. In the second step, each of the other work-related variables was entered one at a time. If the inclusion of a potential confounder resulted in a change in the OR of $\geq 10 \%$, that variable was treated as a real confounder in the multiple regression models. Respondents from different occupational settings (ie, the general working population) were included to obtain a contrast in the work-related exposures studied. Hence, occupational group was not introduced as a confounder in the regression models in order to avoid over adjustment. Interactions between exposures of interest and confounder variables were not tested due to the low number of cases and the lack of power to detect possible interaction effects. All statistical analyses were conducted with SPSS Statistics for Windows, version 20.0 (IBM Corporation, Armonk, NY, USA).

For statistically significant work-related factors in the regression analyses, we calculated the population attributable risk (PAR) estimates with 95\% CI (table $3)$. In contrast to OR estimates, the PAR estimate combines data on prevalence and a measure of association to provide a quantitative estimate of the proportion of cases in the population that is attributable to a particular exposure. Based on the method described by Natarajan et al (27), PAR was calculated using the formula $[\mathrm{Pd} \times((\mathrm{OR}-1) / \mathrm{OR})]$, where $\mathrm{Pd}$ is the proportion of cases (ie, work disability at follow-up) exposed to a risk factor. The lower and upper limits of the 95\% CI for PAR were calculated from the general PAR formula using the lower and upper limits of the $97.5 \%$ CI for $\mathrm{Pd}$ and OR. This constitutes the $95 \%$ Bonferroni CI for PAR. Based on the assumption that the model variables are statistically independent and there are no interac- 
Table 1. Distribution of the sociodemographic and health variables at baseline and the associated risk of work disability at the 3-year follow-up. [95\% Cl=95\% confidence interval]

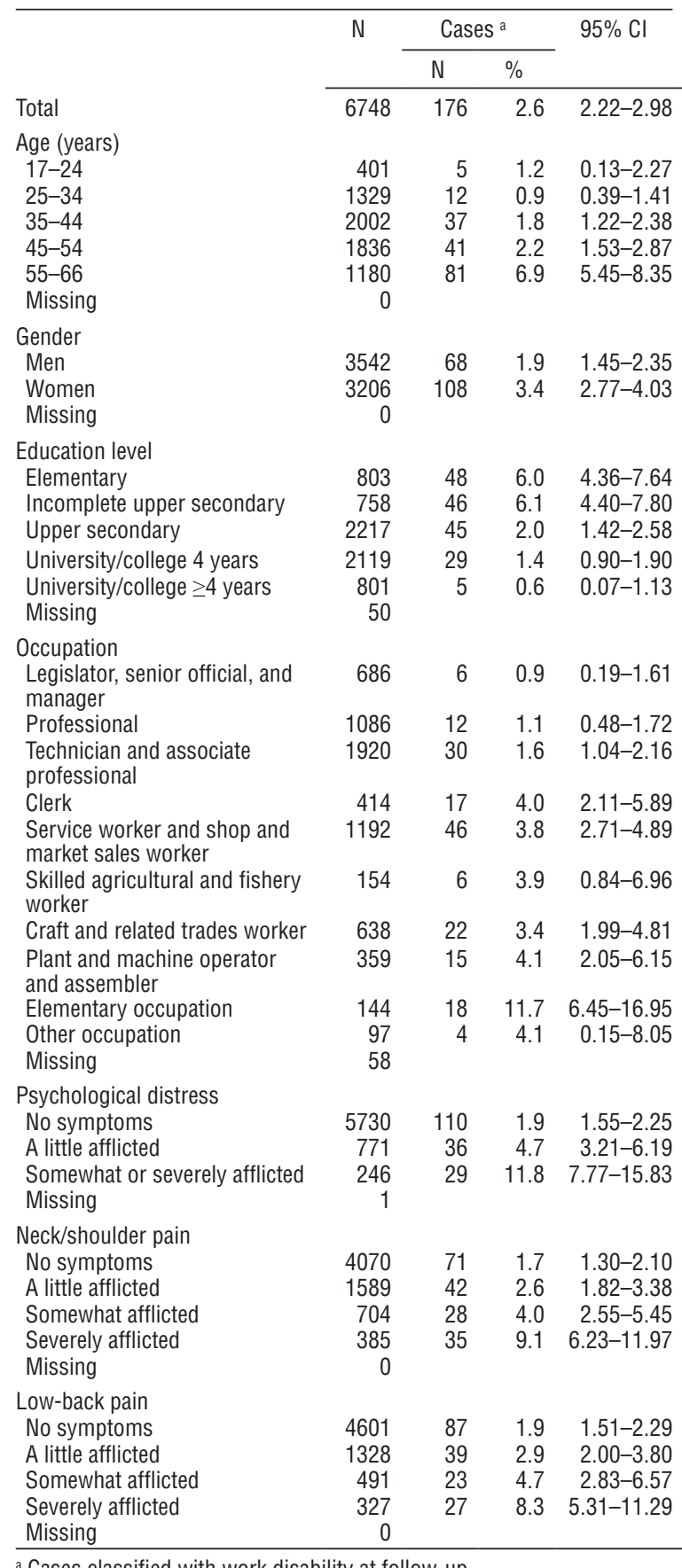

${ }^{a}$ Cases classified with work disability at follow-up.

tions in the logistic model, the summary attributable risk was calculated according to the formulae: $1-\left(1-\mathrm{PAR}_{\mathrm{var1}}\right)$ $\left(1-\mathrm{PAR}_{\text {var2 }}\right)\left(1-\mathrm{PAR}_{\text {var3 }}\right)$ etc $\ldots$ (28).

\section{Results}

Table 1 describes the distribution of sociodemographic and health variables at baseline and the associated risk of work disability at the follow-up. In total, 2.6\% (176 individuals) were classified as having work disability during the follow-up. The risk increased with age and was higher among women than men $(3.4 \%$ versus $1.9 \%$, respectively) and among employees with less education ( $6 \%$ for basic school level versus $0.6 \%$ for $\geq 4$ years of university/college). Moreover, work disability was related to the severity of psychological distress symptoms and musculoskeletal pain. Average weekly working hours at baseline was lower among work disability cases than non-cases [mean 32.5, standard deviation (SD) 11.9 and 38.3, SD 10.6, respectively].

Table 2 shows the results of multiple logistic analyses with baseline psychosocial and mechanical exposure as the predictors and work disability at follow-up as the outcome. A low level of supportive leadership, bullying/harassment, and monotonous work predicted work disability in model 1 (adjusted for age and sex). Estimates ranged from OR 2.20 (monotonous work) to OR 2.93 (bullying/harassment). In the fully adjusted model 2, the effects of low supportive leadership and monotonous work were reduced but remained borderline significant predictors (OR 1.61, 95\% CI 1.02-2.56 and OR 1.53, 95\% CI 1.09-2.16, respectively). By contrast, the effect of bullying/harassment was no longer significant when adjusted for health complaints; the overall OR declined by $35 \%$, mainly because of a $26 \%$ decrease when adjusted for psychological distress. No mechanical factors acted as confounders related to any of the psychosocial factors (correlations between psychosocial and mechanical variables ranged between $\mathrm{r}=0.06$ and $\mathrm{r}=0.15$ ).

All mechanical risk factors evaluated were significant predictors of work disability in model 1 (adjusted for age and sex). Estimates ranged from OR 1.87 (squatting/kneeling) to OR 5.90 (whole-body vibration). All risk estimates were reduced substantially when adjusted for educational level and ranged from a $12 \%$ reduction for neck flexion to a $35 \%$ reduction for heavy lifting. A further reduction was observed when adjusted for health complaints; the reductions ranged from $4 \%$ for whole-body vibration to $14 \%$ for awkward lifting. In the fully adjusted model 2 , the significant predictors were neck flexion ( $\geq 3 / 4$ of the workday: OR 2.49, $95 \%$ CI 1.36-4.56), standing ( $\geq 3 / 4$ of the workday: OR 1.79 , 95\% CI 1.21-2.65) and whole-body vibration $(\geq 3 / 4$ of the workday: OR 4.15, 95\% CI 1.77-9.71). There was some internal confounding between the mechanical dimensions. Awkward lifting was no longer significant after adjusting for standing at work $(\geq 1 / 4$ of the workday: 
Table 2. Multiple logistic regression: work disability at the 3-year follow-up, work-regressed on work-related exposures measured at baseline. [OR=0dds ratio; $95 \% \mathrm{Cl}=95 \%$ confidence interval.]

\begin{tabular}{|c|c|c|c|c|c|c|c|}
\hline & \multirow[t]{2}{*}{ N } & \multirow[t]{2}{*}{ Cases $^{\text {a }}$} & \multirow[t]{2}{*}{$\%$ a } & \multicolumn{2}{|c|}{ Model $1^{\text {b }}$} & \multicolumn{2}{|c|}{ Model $2^{c}$} \\
\hline & & & & OR & $95 \% \mathrm{Cl}$ & $\mathrm{OR}$ & $95 \% \mathrm{Cl}$ \\
\hline Job demands (ref=low) & 2528 & 79 & 3.1 & 1.00 & & 1.00 & \\
\hline Medium & 676 & 10 & 1.5 & 0.48 & $0.25-0.94$ & 0.59 & $0.30-1.18$ \\
\hline High & 1809 & 35 & 1.9 & 0.69 & $0.46-1.04$ & 0.82 & $0.53-1.26$ \\
\hline Missing & 1735 & 52 & & & & & \\
\hline Job control (ref=high) & 2556 & 69 & 2.7 & 1.00 & & 1.00 & \\
\hline Medium & 2680 & 58 & 2.2 & 0.80 & $0.56-1.15$ & 0.79 & $0.55-1.15$ \\
\hline Low & 1085 & 39 & 3.6 & 1.28 & $0.85-1.95$ & 0.84 & $0.54-1.30$ \\
\hline Missing & 427 & 10 & & & & & \\
\hline Supportive leadership (ref=high) & 4367 & 101 & 2.3 & 1.00 & & 1.00 & \\
\hline Medium & 1324 & 33 & 2.5 & 0.99 & $0.66-1.49$ & 0.85 & $0.56-1.29$ \\
\hline Low & 528 & 28 & 5.3 & 2.24 & $1.45-3.47$ & 1.61 & $1.02-2.56$ \\
\hline Missing & 529 & 14 & & & & & \\
\hline Bullying/harassment (ref=no) & 4487 & 108 & 2.4 & 1.00 & & $1.00^{d}$ & \\
\hline Yes & 264 & 14 & 5.3 & 2.93 & $1.63-5.27$ & 1.52 & $0.78-2.93$ \\
\hline Missing & 1997 & 54 & & & & & \\
\hline Monotonous work (ref=no) & 3443 & 65 & 1.9 & 1.00 & & 1.00 & \\
\hline Medium & 759 & 13 & 1.7 & 1.00 & $0.55-1.83$ & 0.91 & $0.49-1.69$ \\
\hline High & 2522 & 98 & 3.9 & 2.20 & $1.59-3.04$ & 1.53 & $1.09-2.16$ \\
\hline Missing & 24 & 0 & & & & & \\
\hline Neck flexion (ref=no) & 2515 & 58 & 2.3 & 1.00 & & 1.00 & \\
\hline Exposed $1 / 4$ of the work-day & 340 & 3 & 0.9 & 0.38 & $0.12-1.24$ & 0.34 & $0.10-1.12$ \\
\hline Exposed $1 / 2$ of the work-day & 252 & 13 & 5.2 & 1.93 & $1.03-3.62$ & 1.47 & $0.76-2.84$ \\
\hline Exposed $3 / 4$ of the work-day & 223 & 17 & 7.6 & 3.23 & $1.82-5.72$ & 2.49 & $1.36-4.56$ \\
\hline Missing & 3418 & 85 & & & & & \\
\hline Awkward lifting (ref=no) & 5775 & 132 & 2.3 & 1.00 & & $1.00^{e}$ & \\
\hline Exposed $1 / 4$ of the work-day & 602 & 30 & 5.0 & 2.75 & $1.82-4.17$ & 1.75 & $1.12-2.73$ \\
\hline Exposed $1 / 2$ of the work-day & 249 & 13 & 5.2 & 2.77 & $1.53-5.03$ & 1.35 & $0.70-2.59$ \\
\hline Exposed $3 / 4$ of the work-day & 114 & 1 & 0.9 & 0.52 & $0.07-3.76$ & 0.26 & $0.03-1.91$ \\
\hline Pooled estimate ${ }^{f}$ & & & & 2.51 & $1.76-3.59$ & 1.44 & $0.97-2.14$ \\
\hline Missing & 8 & 0 & & & & & \\
\hline Hands above shoulder $(r e f=n o)$ & 5818 & 141 & 2.4 & 1.00 & & 1.00 & \\
\hline Exposed $1 / 4$ of the work-day & 472 & 20 & 4.2 & 2.28 & $1.40-3.71$ & 1.57 & $0.94-2.61$ \\
\hline Exposed $1 / 2$ of the work-day & 283 & 6 & 2.1 & 1.04 & $0.45-2.40$ & 0.68 & $0.29-1.61$ \\
\hline Exposed $3 / 4$ of the work-day & 166 & 9 & 5.4 & 2.63 & $1.25-5.56$ & 1.67 & $0.77-3.62$ \\
\hline Pooled estimate ${ }^{f}$ & & & & 1.93 & $1.31-2.85$ & 1.29 & $0.86-1.94$ \\
\hline Missing & 9 & 0 & & & & & \\
\hline Squatting/kneeling (ref=no) & 5661 & 138 & 2.4 & 1.00 & & $1.00^{g}$ & \\
\hline Exposed $1 / 4$ of the work-day & 637 & 27 & 4.2 & 2.09 & $1.35-3.23$ & 1.18 & $0.72-1.95$ \\
\hline Exposed $1 / 2$ of the work-day & 298 & 8 & 2.7 & 1.57 & $0.76-3.27$ & 0.87 & $0.40-1.90$ \\
\hline Exposed $3 / 4$ of the work-day & 147 & 3 & 2.0 & 1.30 & $0.40-4.19$ & 0.73 & $0.21-2.46$ \\
\hline Pooled estimate ${ }^{f}$ & & & & 1.87 & $1.28-2.72$ & 1.05 & $0.67-1.63$ \\
\hline Missing & 5 & 0 & & & & & \\
\hline Standing $(r e f=n o)$ & 2890 & 44 & 1.5 & 1.00 & & 1.00 & \\
\hline Exposed $1 / 4$ of the work-day & 566 & 15 & 2.7 & 1.76 & $0.95-3.25$ & 1.29 & $0.69-2.43$ \\
\hline Exposed $1 / 2$ of the work-day & 1047 & 32 & 3.1 & 2.23 & $1.40-3.55$ & 1.77 & $1.10-2.85$ \\
\hline Exposed $3 / 4$ of the work-day & 2234 & 85 & 3.8 & 2.63 & $1.81-3.84$ & 1.79 & $1.21-2.65$ \\
\hline Missing & 11 & 0 & & & & & \\
\hline Heavy lifting (ref=no) & 5319 & 130 & 2.4 & 1.00 & & $1.00^{\mathrm{e}}$ & \\
\hline $1-4$ & 628 & 19 & 3 & 1.53 & $0.93-2.50$ & 0.96 & $0.57-1.62$ \\
\hline $5-19$ & 526 & 16 & 3 & 1.52 & $0.87-2.64$ & 0.94 & $0.52-1.69$ \\
\hline$\geq 20$ & 268 & 10 & 3.7 & 2.15 & $1.06-4.37$ & 0.97 & $0.46-2.06$ \\
\hline Missing & 7 & 1 & & & & & \\
\hline Whole-body vibration (ref=no) & 6489 & 164 & 2.5 & 1.00 & & 1.00 & \\
\hline Exposed $1 / 4$ of the work-day & 99 & 3 & 3 & 1.74 & $0.53-5.69$ & 1.09 & $0.32-3.71$ \\
\hline Exposed $1 / 2$ of the work-day & 63 & 2 & 3.2 & 2.12 & $0.50-9.00$ & 1.51 & $0.35-6.52$ \\
\hline Exposed $3 / 4$ of the work-day & 93 & 7 & 7.5 & 5.90 & $2.59-13.47$ & 4.15 & $1.77-9.71$ \\
\hline Missing & 4 & 0 & & & & & \\
\hline Heavy physical work (ref=no) & 5786 & 136 & 2.4 & 1.00 & & $1.00 \mathrm{e}$ & \\
\hline Exposed $1 / 4$ of the work-day & 567 & 19 & 3.4 & 2.03 & $1.17-3.17$ & 1.04 & $0.61-1.78$ \\
\hline Exposed $1 / 2$ of the work-day & 246 & 10 & 4.1 & 2.08 & $1.03-4.19$ & 1.14 & $0.55-2.34$ \\
\hline Exposed $3 / 4$ of the work-day & 138 & 11 & 8 & 5.38 & $2.76-10.48$ & 2.23 & $1.08-4.57$ \\
\hline Missing & 11 & & & & & & \\
\hline
\end{tabular}

a Cases classified with work disability at follow-up.

${ }^{\mathrm{b}}$ Adjusted for gender and age.

${ }^{c}$ Adjusted for gender and age + education level, psychological distress, neck/shoulder pain and low-back pain and work-related mechanical and/or psychosocial exposures yielding a 10\% change of OR (shown in superscript after the reference value in Model 2).

${ }^{a}$ Adjusted for supportive leadership.

${ }^{\mathrm{e}}$ Adjusted for standing.

$\mathrm{f} \geq \mathrm{1} / \mathrm{4}$ of the workday.

${ }^{9}$ Adjusted for awkward lifting and standing. 
Table 3. Calculated population attributable risk (PAR) based on the statistically significant odds ratio from Models 1 and 2 in table 2. [ $95 \% \mathrm{Cl}=95 \%$ confidence interval. $]$

\begin{tabular}{|c|c|c|c|c|}
\hline & \multicolumn{2}{|r|}{ Model 1} & \multicolumn{2}{|c|}{ Model 2} \\
\hline & PAR & $95 \% \mathrm{Cl}$ & PAR & $95 \% \mathrm{Cl}$ \\
\hline $\begin{array}{l}\text { Low level of supportive } \\
\text { leadership }\end{array}$ & 9.57 & $2.84-17.45$ & 6.55 & $-0.53-15.17$ \\
\hline Bullying/harassment & 7.55 & $1.66-14.81$ & . & \\
\hline $\begin{array}{l}\text { Monotonous work (exposed } \\
3 / 4 \text { of the workday) }\end{array}$ & 30.37 & $17.73-40.82$ & 19.29 & $1.80-33.26$ \\
\hline $\begin{array}{l}\text { Neck flexion (exposed } 3 / 4 \text { of } \\
\text { the workday) }\end{array}$ & 12.90 & $3.85-23.35$ & 11.17 & $1.86-22.24$ \\
\hline $\begin{array}{l}\text { Awkward lifting (exposed } \\
\geq 1 / 4 \text { of the workday) }\end{array}$ & 15.04 & $7.11-23.76$ & · & \\
\hline $\begin{array}{l}\text { Squatting/kneeling (exposed } \\
\geq 1 / 4 \text { of the workday) }\end{array}$ & 10.03 & $2.57-18.60$ & 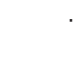 & \\
\hline $\begin{array}{l}\text { Standing (exposed } 3 / 4 \text { of the } \\
\text { workday) }\end{array}$ & 29.93 & $18.50-39.24$ & 21.28 & $5.24-33.32$ \\
\hline $\begin{array}{l}\text { Heavy lifting ( } 20 \mathrm{~kg} \geq 20 \\
\text { times/day) }\end{array}$ & 3.06 & $0.30-14.84$ & . & \\
\hline $\begin{array}{l}\text { Whole-body vibration (exposed } \\
3 / 4 \text { of the workday) }\end{array}$ & 3.30 & $0.38-6.80$ & 3.02 & $0.25-4.51$ \\
\hline $\begin{array}{l}\text { Heavy physical work (exposed } \\
3 / 4 \text { of the workday) }\end{array}$ & 5.09 & $1.30-9.44$ & 3.45 & $-0.05-8.30$ \\
\hline
\end{tabular}

OR $1.44,95 \%$ CI $0.97-2.14)$. Squatting/kneeling was no longer significant after adjusting for standing and awkward lifting. Finally, there was no increased risk associated with heavy lifting after adjusting for standing at work. No psychosocial factors acted as confounders related to any of the mechanical factors.

The estimated PAR of work disability due to workrelated psychosocial and mechanical factors is shown in table 3. Based on the partially adjusted model 1 (table 2), statistically significant PAR estimates were found for low supportive leadership, bullying /harassment, monotonous work, neck flexion, awkward lifting, squatting/kneeling, standing, heavy lifting, and whole-body vibration. Based on the fully adjusted estimates in model 2 (table 2), the highest PAR was estimated for standing ( $3 / 4$ of the workday: PAR $21.28,95 \%$ CI $5.24-33.32$ ) and monotonous work $(3 / 4$ of the workday: PAR $19.29,95 \%$ CI 1.80-33.26). Other statistically significant mechanical factors were neck flexion and whole-body vibration. About $45 \%$ of the disability cases were attributable to these work-related exposures.

\section{Discussion}

This study investigated the role of both psychosocial and mechanical factors at work as risk factors for work disability over a 3-year period in the general working population. Work disability rates were increased among women, older employees, employees with fewer years of education, and employees with higher levels of psychological distress and musculoskeletal complaints. After adjusting for these factors, we estimated that about $45 \%$ of the disability cases were attributable to workrelated exposure. Significant work-related psychosocial risk factors were low level of supportive leadership and monotonous work and mechanical factors such as prolonged standing, neck flexion, whole-body vibration, and heavy physical work. Interventions aimed at reducing work disability in the general working population may benefit from focusing on a both work-related psychosocial and mechanical risk factors.

The strengths of this study are that it was a large nationwide study using random sampling, it used a prospective design with a comprehensive set of exposures measured, it included a thorough control of confounding factors, and it had a high response rate and a large sample size. Even though a substantial percentage (33\%) did not respond at baseline, those who responded to the survey at baseline did not differ systematically from those who did not respond across the benchmarks of age, sex and region. Moreover, in additional analyses, we compared the baseline and the follow-up samples and found no reason to suspect any exposure or outcome bias due to dropout from baseline to follow-up, at least not to the extent that it substantially impacted the observed results. On the other hand, we do not know whether people with poor health were less likely to respond at baseline, which may have led to biased and attenuated estimates and, thus, threatened the internal validity. Moreover, all data were collected by self-report. Hence, bias due to imprecision in the measurement of covariates and outcome cannot be ruled out. It is also possible that the association between self-reported exposure and disability is confounded by the baseline health status of the respondent. Although adjusting for symptoms of psychological distress and musculoskeletal pain at baseline should reduce the risk of reporting bias, this cannot be excluded. On the other hand, because work disability in the present study was based on self-reported illness or disease, it is probable that the effect of workrelated factors on work disability is mediated by poor health. The attenuated association between self-assessed exposure and work disability after adjustment for health indicators may thus reflect this mediator effect rather than health acting as a confounder. Finally, most studies of work disability use administrative data (ie, disability pension), which may reflect doctor and patient attributions, and non-medical factors such as welfare regimes and unemployment (29). The outcome in the present study was self-reported withdrawal from work because of own ill-health and/or disease. Unfortunately we were not able to make a distinction between a temporary and permanent work disability. A similar definition of work disability has, however, been applied in other studies $(8,30)$ and could be considered a robust indicator of 
health-related work disability independent of differences in national social security systems.

Consistent with most previous studies, our study investigated the importance of the core dimensions of the job-demand-control (social support) model as risk factors for work disability. A low level of supportive leadership was the only predictor of work disability in the present study, an observation that is consistent with the findings of a study of the general working population in Finland $(8,17)$. As in other general working population studies, we found that high job demand was not a significant predictor $(6,12,15)$. By contrast, the finding that low decision control was not associated with work disability is contradictory to findings of previous general working population studies from Finland (31), Denmark (10), and Norway (11). However, there are studies that did not find an increased risk associated with low job control $(31,32)$.

Bullying/harassment was associated with a 3-fold increased risk for work disability in the partly adjusted model, but was no longer a significant predictor after adjusting for psychological distress. Psychological distress may be considered a mediating factor between exposure to bullying and risk of work disability. However, some studies have indicated that psychological distress may also be a risk factor for bullying (33) and work disability (19).

Monotonous work was the most important psychosocial factor in the present study and has been reported as a risk factor in general working population studies from Finland, Denmark, and the UK $(10,34,35)$. We estimated that about $11 \%$ of the work disability cases could be attributed to monotonous and repetitive work, a value that is slightly lower than the $13 \%$ and $23 \%$ among men and women, respectively, reported in a study from Denmark (3). Conversely, another study from Denmark reported an inverse relation between variation in work and work disability (10).

The two most important risk factors for work disability at the population level in the present study were prolonged standing and neck flexion: $21.3 \%$ and $11.2 \%$ of all disability cases were attributable to these working conditions, respectively. Prolonged standing has some support in the literature as a risk factor for work disability and reduced work ability $(6,36)$, whereas neck flexion has been shown to increase the risk of neck pain and sick leave associated with neck pain $(37,38)$.

The strongest associations in the present study were observed for two relatively low-prevalence exposures: whole-body vibration and heavy physical work. However, because of the small number of cases, the confidence intervals were wide. The percentage of disability cases attributed to whole-body vibration was similar to that estimated in a general working-population study of men in Denmark (3\% versus 5\%, respectively) (7), whereas the percentage of cases attributed to heavy physical work was lower than that reported in the general working population in Denmark (3\% versus $8 \%$, respectively) (3). No consistent significant associations with work disability were observed for the other mechanical factors assessed in the present study.

In conclusion, this study provides evidence of a significant relationship between both work-related mechanical and psychosocial factors and self-reported work disability in the general working population. Interventions aimed at reducing retirement from work because of illness and/or disease in the general working population may benefit from focusing on monotonous jobs, low levels of supportive leadership, and physically demanding jobs involving prolonged standing, whole-body vibration, and neck flexion. Interventions directed at highly exposed individuals are likely to have the largest impact.

The author declares no conflicts of interest.

\section{References}

1. OECD. Key Trends and Outcomes in Sickness and Disability, OECD Publishing; 2010.

2. Stattin M, Jarvholm B. Occupation, work environment, and disability pension: a prospective study of construction workers. Scand J Public Health. 2005;33:84-90. http://dx.doi. org/10.1080/14034940410019208.

3. Labriola M, Feveile H, Christensen KB, Stroyer J, Lund T. The impact of ergonomic work environment exposures on the risk of disability pension: Prospective results from DWECS/ DREAM. Ergonomics. 2009;52:1419-22. http://dx.doi. org/10.1080/00140130903067771.

4. Lahelma E, Laaksonen M, Lallukka T, Martikainen P, Pietilainen O, Saastamoinen P, et al. Working conditions as risk factors for disability retirement: a longitudinal register linkage study. BMC Public Health. 2012;12:309-18. http:// dx.doi.org/10.1186/1471-2458-12-309.

5. Hagen KB, Tambs K, Bjerkedal T. A prospective cohort study of risk factors for disability retirement because of back pain in the general working population. Spine. 2002;27:1790-6. http://dx.doi.org/10.1097/00007632-200208150-00019.

6. Albertsen K, Lund T, Christensen KB, Kristensen TS, Villadsen E. Predictors of disability pension over a 10 -year period for men and women. Scandinavian Journal of Public Health. 2007;35:78-85. http://dx.doi. org/10.1080/14034940600858474.

7. Tuchsen F, Feveile H, Christensen KB, Krause N. The impact of self-reported exposure to whole-body-vibrations on the risk of disability pension among men: a 15 year prospective study. BMC Public Health. 2010;10:305-10. http://dx.doi. org/10.1186/1471-2458-10-305.

8. Krause N, Lynch J, Kaplan GA, Cohen RD, Goldberg DE, 
Salonen JT. Predictors of disability retirement. Scand J Work Environ Health. 1997;23:403-13. http://dx.doi.org/10.5271/ sjweh.262.

9. Allebeck P, Mastekaasa A. Swedish Council on Technology Assessment in Health Care (SBU). Chapter 5. Risk factors for sick leave - general studies. Scand J Public Health. Suppl 2004;63:49-108. http://dx.doi. org/10.1080/14034950410021853.

10. Christensen KB, Feveile H, Labriola M, Lund T. The impact of psychosocial work environment factors on the risk of disability pension in Denmark. Eur J Public Health. 2008;18:235-. http://dx.doi.org/10.1093/eurpub/ckm130.

11. Krokstad S, Johnsen R, Westin S. Social determinants of disability pension: a 10-year follow-up of 62000 people in a Norwegian county population. Int J Epidemiol. 2002;31:118391. http://dx.doi.org/10.1093/ije/31.6.1183.

12. Blekesaune M, Solem PE. Working conditions and early retirement: A prospective study of retirement behavior. Research on Aging. 2005;27:3-30. http://dx.doi. org/10.1177/0164027504271438.

13. Robroek SJ, Schuring M, Croezen S, Stattin M, Burdorf A. Poor health, unhealthy behaviors, and unfavorable work characteristics influence pathways of exit from paid employment among older workers in Europe: a four year follow-up study. Scand J Work Environ Health. 2013 Mar 1;39(2):125-33. http://dx.doi.org/10.5271/sjweh.3319.

14. Samuelsson A, Ropponen, A, Alexanderson K, Svedberg P. Psychosocial working conditions, occupational groups, and risk of disability pension due to mental diagnoses: a cohort study of 43000 Swedish twins. Scand J Work Environ Health. 2012 Dec 17. [Epub ahead of print] http://dx.doi.org/10.5271/ sjweh.3338.

15. Laine S, Gimeno D, Virtanen M, Oksanen T, Vahtera J, Elovainio M, et al. Job strain as a predictor of disability pension: the Finnish Public Sector Study. J. Epidemiol. Community Health. 2009;63:24-30. http://dx.doi. org/10.1136/jech.2007.071407.

16. Mantyniemi A, Oksanen T, Salo P, Oksanen T, Vahtera J, Elovainio M, et al. Job strain and the risk of disability pension due to musculoskeletal disorders, depression or coronary heart disease: a prospective cohort study of 69842 employees. Occup Environ Med. 2012;69:574-81. http://dx.doi. org/10.1136/oemed-2011-100411.

17. Sinokki M, Hinkka K, Ahola K, Gould R, Puukka P, Lonnqvist J, et al. Social support as a predictor of disability pension: the Finnish Health 2000 study. J Occup Environ Med. 2010;52:733-9. http://dx.doi.org/10.1097/ JOM.0b013e3181e79525.

18. Labriola M, Lund T. Self-reported sickness absence as a risk marker of future disability pension. Prospective findings from the DWECS/DREAM study 1990-2004. Int J Med Sci. 2007;4:153-8. http://dx.doi.org/10.7150/ijms.4.153.

19. Lund T, Csonka A. Risk factors in health, work environment, smoking status, and organizational context for work disability. Am J Ind Med. 2003;44:492-501. http://dx.doi.org/10.1002/ ajim. 10298.
20. MacDonald LA, Karasek RA, Punnett L, Scharf T. Covariation between workplace physical and psychosocial stressors: evidence and implications for occupational health research and prevention. Ergonomics. 2001;44:696-718.

21. Bang Christensen K, Lund T, Labriola M, Villadsen E, Bultmann U. The fraction of long-term sickness absence attributable to work environmental factors: prospective results from the Danish Work Environment Cohort Study. Occup Environ Med. 2007;64:487-9. http://dx.doi.org/10.1136/ oem.2006.028563.

22. Statistics Norway. Samordnet levekårsundersøkelse 2009 -Tverrsnitt. Tema: Arbeidsmiljø [Coordinated Living Conditions Survey 2009 - Cross Sectional. Focus: Work environment).[Report in Norwegian]. Statistics Norway, Oslo; 2010.

23. Sterud T, Tynes T. Work-related psychosocial and mechanical risk factors for low back pain: a 3-year follow-up study of the general working population in Norway. Occup Environ Med. 2013 Jan 15 [Epub ahead of print]. http://dx.doi.org/10.1136/ oemed-2012-101116.

24. Dallner M. Validation of the General Nordic Questionnaire (QPSNordic) for psychological and social factors at work, Nordisk Ministerråd, Copenhagen; 2000.

25. Orhede E. Nordic cooperation in research on the work environment. Scand J Work Environ Health. 1994;20:65-66. http://dx.doi.org/10.5271/sjweh.1426.

26. Rothman KJ, Greenland S, Lash TL. Modern epidemiology, Lippincott Williams \& Wilkins, Philadelphia; 2008.

27. Natarajan S, Lipsitz SR, Rimm E. A simple method of determining confidence intervals for population attributable risk from complex surveys. Stat Med. 2007;26:3229-39. http://dx.doi.org/10.1002/sim.2779.

28. Bruzzi P, Green SB, Byar DP, Brinton LA, Schairer C. Estimating the population attributable risk for multiple risk factors using case-control data. Am J Epidemiol. 1985;122(5):904-14

29. Stattin M. Retirement on grounds of ill health. Occup Environ Med. 2005;62:135-40. http://dx.doi.org/10.1136/ oem.2004.015669.

30. Vahtera J, Laine S, Virtanen M, Oksanen T, Koskinen A, Pentti J, et al. Employee control over working times and risk of cause-specific disability pension: the Finnish Public Sector Study. Occup Environ Med. 2010;67:479-85. http://dx.doi. org/10.1136/oem.2008.045096.

31. Alavinia SM, de Boer AGEM, van Duivenbooden JC, FringsDresen MHW, Burdorf A. Determinants of work ability and its predictive value for disability. Occupational Medicine (Oxford). 2009;59:32-7. http://dx.doi.org/10.1093/occmed/ kqn148.

32. Lund T, Iversen L, Poulsen KB. Work environment factors, health, lifestyle and marital status as predictors of job change and early retirement in physically heavy occupations. Am J Ind Med. 2001;40:161-9. http://dx.doi.org/10.1002/ajim.1084.

33. Finne LB, Knardahl S, Lau B. Workplace bullying and mental distress - a prospective study of Norwegian employees. Scand 
J Work Environ Health. 2011;37:276-87. http://dx.doi. org/10.5271/sjweh.3156.

34. Appelberg K, Romanov K, Heikkila K, Honkasalo ML, Koskenvuo M. Interpersonal conflict as a predictor of work disability: a follow-up study of 15,348 Finnish employees. J Psychosom Res. 1996;40:157-67. http://dx.doi. org/10.1016/0022-3999(95)00576-5.

35. Pope DP, Croft PR, Pritchard CM, Silman AJ, Macfarlane GJ. Occupational factors related to shoulder pain and disability. Occup Environ Med. 1997;54:316-21. http://dx.doi. org/10.1136/oem.54.5.316.

36. Tuomi K, Ilmarinen J, Martikainen R, Aalto L, Klockars M. Aging, work, life-style and work ability among Finnish municipal workers in 1981-1992. Scand J Work Environ Health. 1997;23 Suppl 1:58-65.

37. Ariens GA, Bongers PM, Douwes M, Miedema MC, Hoogendoorn WE, van der Wal G, et al. Are neck flexion, neck rotation, and sitting at work risk factors for neck pain? Results of a prospective cohort study. Occup Environ Med. 2001;58:200-7. http://dx.doi.org/10.1136/oem.58.3.200.

38. Ariens GA, Bongers PM, Hoogendoorn WE, van der Wal G, van Mechelen W. High physical and psychosocial load at work and sickness absence due to neck pain. Scand J Work Environ Health. 2002;28:222-31. http://dx.doi.org/10.5271/ sjweh.669.

Received for publication: 1 November 2012 